Revue d'histoire de l'Amérique française

REVUE D.HISTOIRE DE L'AMÉRIQUE FRANÇAISE

\title{
Prêtres de Montréal en mission aux États-Unis (1836-1876)
}

\section{François Beaudin}

Volume 21, numéro 4, mars 1968

URI : https://id.erudit.org/iderudit/302725ar

DOI : https://doi.org/10.7202/302725ar

Aller au sommaire du numéro

Éditeur(s)

Institut d'histoire de l'Amérique française

ISSN

0035-2357 (imprimé)

1492-1383 (numérique)

Découvrir la revue

Citer cet article

Beaudin, F. (1968). Prêtres de Montréal en mission aux États-Unis (1836-1876). Revue d'histoire de l'Amérique française, 21(4), 792-802.

https://doi.org/10.7202/302725ar d'utilisation que vous pouvez consulter en ligne.

https://apropos.erudit.org/fr/usagers/politique-dutilisation/ 


\section{PRETRES DE MONTREAAL EN MISSION AUX ÉTATS-UNIS $(\mathbf{1 8 3 6 - 1 8 7 6 ) *}$}

\section{INTRODUCTION}

Le chanoine Lionel Groulx, (grand historien du Québec), dont nous saluons la mémoire ici avec vous, a intitulé un de ses ouvrages: "Le Canada français missionnaire". Cet ouvrage, paru chez Fides, à Montréal, en 1962, se divise en deux parties: les missions canadiennes et les missions à l'extérieur de l'Amérique du Nord. A première vue, cette division même laisserait croire que l'Église canadienne n'a rien eu à voir avec la fondation et le progrès de l'Église catholique aux États-Unis. Mais détrompons-nous. Ce qui a empêché le chanoine Groulx de traiter ce sujet, c'est, à notre avis, le trop petit nombre de monographies fouillées sur ces missions. Ne nous dit-il pas lui-même, dès les premières pages de son Canada français missionnaire: "[Le petit peuple canadien-français], dès le début du XIX's., s'élance à la conquête de l'Ouest jusqu'aux rives du Pacifique; il pousse son aventure à l'Extrême-Nord, y atteint les régions de l'Arctique. Entre-temps, il donne à l'Église de maints États de la république américaine l'on ne sait combien de ses fils et de ses filles." ${ }^{1}$

C'est afin d'apporter notre part à l'élaboration de l'histoire de l'Église canadienne dans ses rapports avec les diocèses américains que nous avons accepté l'offre, transmise par le Centre de Recherches en Histoire religieuse canadienne de l'Université St-Paul d'Ottawa, de vous entretenir de la contribution du diocèse de Montréal à l'œuvre des missions aux États-Unis. Nous le ferons en nous limitant à l'apport en prêtres séculiers fourni par ce diocèse. Nous limiterons aussi notre

* Communication présentée, à titre de membre de la Commission consultative du Centre de recherches en histoire religieuse, de l'Université St-Paul d'Ottawá, le 30 décembre 1967.

1 Chan. Lionel Groulx, Le Canada français missionnaire (Fides, Montréal et Paris, 1962), 9. 
recherche à la période 1836-1876. Il faudra, dans l'avenir, pour donner une idée complète de l'œuvre gigantesque accomplie aux Etats-Unis par le diocèse de Montréal et le Canada français en général de nombreuses monographies semblables à celle-ci. Elles devront porter sur l'œuvre des autres diocèses, sur les communautés religieuses de Pères, de Frères et de Sœurs, et s'étendre sur des périodes plus vastes. Car nous sommes portés à croire que la contribution décrite ici n'a fait qu'augmenter jusqu'à la guerre de 1914.

\section{SECTEUR DE RECHERCHE CHOISI}

La recherche dont nous vous présentons les résultats a été faite aux Archives de la Chancellerie de l'Archevêché de Montréal (ACAM). Elle porte tant sur les prêtres qui faisaient partie du diocèse de Québec et qui sont devenus prêtres du diocèse de Montréal, au moment de l'érection de celui-ci en 1836, que sur les prêtres ordonnés pour le diocèse de Montréal entre 1836 et 1876, cette dernière date étant choisie à cause de la démission, cette année-là, de $\mathrm{Mgr}$ Ignace Bourget, $2^{e}$ évêque de Montréal.

\section{MÉTHODE}

Pour ce qui est des prêtres de la lère catégorie qu'on appellera les prêtres fondateurs, la liste utilisée, la seule connue à ce jour, est celle qui a paru dans "The Quebec Almanack, and British American Royal Kalendar, for the year 1837", imprimé et vendu par Samuel Neilson, à Québec, 14 Mountain Street. Nous avons travaillé sur une photocopie des pages 148 à 155 incl. de l'original conservé à la Salle Gagnon de la Bibliothèque Municipale de Montréal (Cette photocopie est conservée à nos Archives au dossier 401.001 - Liste du clergé du diocèse de Montréal - 1837).

Pour ce qui est des prêtres ordonnés entre 1836 et 1876 , nous avons fait nous-mêmes, aux Archives, un relevé de tous les prêtres ordonnés pour le diocèse de Montréal entre 1836 et 1965. Nous nous sommes servis pour cette recherche-ci de la tranche 1836-1876 incl. de ce relevé (Ce relevé est conservé 
à nos Archives sous la cote RCD 138). Pour chacun de ces prêtres, nous avons retrouvé l'endroit où est conservé l'enregistrement de l'acte d'ordination, la plupart du temps dans nos propres Archives.

Puis, nous avons repéré, dans Allaire ${ }^{2}$ ou Tanguay ${ }^{3}$, les prêtres appartenant au diocèse de Montréal, d'après les deux listes établies précédemment, qui ont fait un séjour aux ÉtatsUnis pour fins de ministère.

\section{SOMMAIRE}

Nous donnerons donc successivement les fruits de nos recherches sur les points suivants: 1 ) Nombre absolu des prêtres de toute cette période; 2 ) nombre relatif des prêtres fondateurs et des prêtres ordonnés après 1836 ; 3) nombre absolu de ceux qui sont allés aux États-Unis; 4) nombre relatif dans chacun des deux groupes; 5) proportion par rapport au nombre absolu et aux nombres relatifs; 6) répartition géographique des endroits de mission aux Etats-Unis, avec nombre de prêtres de Montréal par États; 7) nombre absolu de prêtres de Montréal en service aux États-Unis, par cinq ans; 8) prêtres ayant joué un rôle important; 9) répartition des prêtres selon l'année d'ordination et l'âge au départ; 10) répartition des prêtres selon le nombre d'années de ministère aux É.-U. et moyenne. 11) Nous terminerons en nous posant quelques questions sur les motifs des prêtres qui partent et sur les motifs de $\mathrm{M}^{\mathrm{gr}}$ Bourget qui les laisse partir.

1) Nombre absolu des prêtres de toute cette période 542

2) Nombre relatif des prêtres fondateurs et des prêtres ordonnés après 1836

Prêtres fondateurs: 114

Prêtres ordonnés après 1836 : 428

2 J.-B.-A. Allaire, ptre, Dictionnaire biographique dw Clergé canadienfrançais (Montréal, Imprimerie de l'Ecole catholique des Sourds-Muets, 1910-1934), 6 vol.

$3 \mathrm{Mgr}$ Cyprien Tanguay, Répertoire Général du Clergé canadien (Montréal, Eusèbe Sénécal et Fils, 1893). 
3) Nombre absolu de ceux qui sont allés aux E.-U. 78

4) Nombre relatif dans chacun des deux groupes Prêtres fondateurs: 9 Prêtres ordonnés après 1836: $\quad 69$

5) Proportion par rapport au nombre absolu et aux nombres relatifs

Par rapport au nombre absolu:

Par rapport aux prêtres fondateurs: $\quad 7 \%$

Par rapport aux prêtres ordonnés après 1836: 16\%

6) Répartition géographique des endroits de mission aux E.-U. avec nombre de prêtres de Montréal par Êtats

Etats Nombre de prêtres

$\begin{array}{lrl}\text { New-York } & 27 & (7) \\ \text { Massachusetts } & 21 & (4) \\ \text { Vermont } & 8 & (3) \\ \text { Oregon } & 7 & (47) \\ \text { New Hampshire } & 6 & (2) \\ \text { Floride } & 3 & (18) \\ \text { Ohio } & 3 & (23)\end{array}$

États où il y a eu deux prêtres de Montréal: Minnesota, Washington, Michigan, Rhode Island, Maryland, Dakota Nord, Illinois.

États où il y a eu un prêtre de Montréal: Texas, Pennsylvania, Indiana, Louisiana, Kentucky, Maine, Missouri, Connecticut, Georgie, Arkansas, Californie.

P.-S. Donc 25 États ont profité de missions de prêtres de Montréal. Noter que certains prêtres ont missionné en plusieurs Etats durant leur séjour, ce qui explique le total de 100 inscriptions sur ce tableau relativement à 78 prêtres qui ont missionné aux É.-U. 
7) Nombre absolu de prêtres de Montréal en ministère aux E.-U. par cinq ans - 1836-1876

1836-1840: 3

1841-1845: 4

1846-1850: 5

1851-1855: 9

1856-1860: 11

1861-1865: 16

1866-1870: 28

1871-1875: 40

Des prêtres fondateurs et des prêtres ordonnés entre 1836 et 1876, on en trouve encore qui sont en service aux EtatsUnis ${ }^{4}$ entre

1876-1880: 39

1881-1885: 34

1886-1890: 25

1891-1895: 19

1896-1900: 17

1901-1905: 11

1906-1910: 6

1911-1915: 1

8) Prêtres ayant joué un rôle important

Rappelons pour mémoire le rôle des deux évêques Elanchet (Augustin-Magloire et François-Norbert), qui étaient des prètres de Montréal; ils ont fondé l'Église dans l'État d'Oregon et de Washington. ${ }^{5}$ En 1862 , la moitié du clergé du diocèse de l'Oregon venait de Montréal.

Rappelons aussi, dans le domaine des missions indiennes, le rôle joué par l'abbé Jean-Baptiste-Abraham Brouillet ou Brouillette. Âgé de 34 ans, après 10 ans d'ordination, il devient, en 1847, missionnaire en Oregon jusqu'en 1860. Cette même année, il est nommé à Washington chef du bureau fédéral des Indiens

${ }^{4}$ ou ne se rendent aux E.-U. qu'à ce moment-là.

5 Voir Clarence B. Bagley, Ed., Early Catholic Missions in Old Oregon, (Seattle, Lowman and Handford Co., 1932), 2 vol. 
catholiques. En 1870, il cumule la charge de grand-vicaire d'Oregon City. En 1878, il deviendra sénateur. Il meurt en $1884 .^{5}$

Enfin, pour donner un exemple de curé bâtisseur, ce que plusieurs de ces prêtres furent, rappelons que l'abbé LouisGuillaume Gagnier en six ans, de 1868 à 1873, fonde neuf paroisses où il bâtit autant d'églises. Il en construira trois autres ensuite, pour mourir en 1908 à Springfield, Mass., dans cette Nouvelle-Angleterre où il s'était tant dévoué.?

9) Áge et nombre d'années de sacerdoce au moment du départ

Pour établir nos données, à ce sujet, nous avions en mains des renseignements pour 73 prêtres sur les 78 qui sont allés aux Etats-Unis.

Pour ce qui est de l'âge, la moyenne d'âge des prêtres, au moment de leur départ pour les États-Unis, s'établit à 35 ans. 41 prêtres sur 73 ont quitté Montréal avant 35 ans; 19 sont partis de Montréal avant d'avoir atteint 30 ans.

On constate donc qu'on a envoyé là des hommes jeunes. Et ça devient encore plus évident quand on regarde le nombre d'années de sacerdoce de ces hommes.

Les 73 prêtres mentionnés plus haut avaient entre 1 et 30 années de sacerdoce au moment du départ. Cependant, 50 d'entre eux se situaient entre 1 et 13 années de sacerdoce, et, parmi eux, 37, soit $50 \%$, se situaient entre 1 et 7 années de sacerdoce. En résumé, 37 ont quitté ayant entre 1 et 7 ans de sacerdoce; 13 avaient entre 8 et 13 années de sacerdoce; 23 , enfin, avaient entre 14 et 30 années de sacerdoce; de ces derniers 17 avaient entre 14 et 24 années de sacerdoce, et 6 avaient entre 25 et 30 années de sacerdoce.

On peut donc en déduire que le diocèse de Montréal a laissé partir pour ces missions américaines un clergé jeune, ce qui est un signe de l'importance que l'on attachait à ces missions, puisque l'on y investissait la fraîche relève.

6 Allaire, 1: 85.

7 Allaire, $6: 294$. 
10) Nombre d'années de ministère aux E.-U. et moyenne

Sur ce point, nous avons des renseignements pour 69 prêtres sur 78.

9 prêtres ont fait aux E.-U. un an de ministère;

27 prêtres ont fait de 2 à 13 ans de ministère;

14 prêtres ont fait de 14 à 24 ans de ministère;

19 prêtres ont fait au-delà de 25 ans de ministère.

Si l'on fait le total, on obtient 1194 années de ministère, et, si l'on fait la moyenne, on obtient $\mathbf{1 7 . 3}$ années. Si l'on enlève les prêtres qui n'ont passé qu'un an, la moyenne passe à 19.7 années. Ce qui est impressionnant.

\section{1) Motifs des prêtres}

Pourquoi tant de prêtres sont-ils allés faire du ministère dans les diocèses américains ou les missions américaines? Voilà la question que l'on peut se poser en terminant cette recherche.

Formulons tout d'abord quelques hypothèses. Qu'est-ce qui attire là-bas? L'esprit d'aventure, un désir d'avancement plus rapide, un climat plus favorable, l'esprit missionnaire, l'appel des évêques américains, le déménagement de la famille du prêtre ou d'amis vers les États-Unis? Qui sait? Peut-être un peu de tout cela.

Mais d'autre part, qu'est-ce qui empêche quelqu'un de rester ici? Des conflits politiques, la mésentente, l'inadaptation ou une certaine saturation des cadres cléricaux?

Enfin pensons surtout à cet évêque de Montréal que fut $\mathbf{M g r}$ Bourget. Il a vraiment, selon le mot de l'Apôtre, porté "le souci de toutes les Églises". À une époque où l'on croyait, sans toujours le formuler, que la langue était gardienne de la foi, il a sûrement regardé comme une contribution au progrès et au maintien de cette foi l'envoi de prêtres parlant français aux groupes francophones des Etats-Unis. Or, il est important de remarquer que sur les 78 prêtres qui sont allés faire du ministère aux États-Unis, 3 seulement étaient de nationalité irlandaise. D'autre part, si l'on additionne les prêtres qui ont fait des stages en Nouvelle-Angleterre, milieu par excellence de l'émigration 
canadienne-française, sur cent séjours différents repérés pour ces 78 prêtres, soixante-six de ces séjours eurent lieu dans des villes de Nouvelle-Angleterre.

Il semble donc qu'à des causes individuelles sont venues se greffer des causes plus générales.

Tâchons de vérifier un peu, par des témoignages, quelquesunes de ces hypothèses.

L'abbé Étienne Chartier, curé de St-Benoit, comté des Deux-Montagnes, près d'Oka, à cinquante milles de Montréal, doit fuir parce qu'il a pris ouvertement position pour les rebelles, lors des troubles de 1837. Son départ a donc comme motif des conflits politiques. Rongé d'ennui, il demandera, de Détroit, en 1845 , son retour à Montréal. ${ }^{8}$

L'abbé Joseph Quevillon, en mission auprès des Canadiens français de Burlington, remercie Mgr Bourget, le 24 août 1850, d'avoir envoyé trois des chanoines de Montréal assister à la bénédiction de la lère pierre de leur église. Il n'était arrivé là que du mois d'avril de la même année. ${ }^{\circ}$ Donc, aide aux émigrés canadiens-français. $M^{\text {gr }}$ de Goësbriand deviendra évêque, en 1853 , à Burlington.

Oeuvre religieuse, la mission de l'Oregon est aussi parce que canadienne œuvre éminemment nationale. Ainsi s'exprime l'abbé Jean-Baptiste-Abraham Brouillet à l'occasion d'une campagne d'aumônes en 1862, avivant ainsi la fierté de ses compatriotes catholiques ... et demandant des efforts et des renforts supplémentaires. Tout cela, d'abord et avant tout pour la conversion des miliers d'Indiens de l'Oregon. ${ }^{10}$

Voici l'exemple typique de l'abbé Isidore Noiseux, rendu à Boston. Il écrit à Mgr Bourget, le 10 février 1857: "La reconnaissance et le devoir m'engagent à vous soumettre les raisons qui m'ont forcé de chercher une place sur une terre étrangère. Votre Grandeur se rappellera qu'étant curé à St-Dominique, je

\footnotetext{
8 ACAM, Dossier 420.048, Lettre du 14 août 1845.

9 ACAM, Dossier 420.056, Lettre du 24 août 1850.

10 ACAM, Dossier 421.009, Imprimé du 30 septembre 1862.
} 
lui demandai la permission d'aller étudier l'anglais dans les Etats-Unis ... Ensuite, quelques circonstances fâcheuses survenues, dans mes affaires temporelles, entre le nouvel évêque de St-Hyacinthe et moi m'ont donné occasion de mettre à exécution les plans que j'avais formés depuis longtemps... Combien d'autres [personnes], même Canadiens, j'ai rencontré dans mes missions, qui n'avaient pas vu de prêtres depuis quinze, vingt ans..." 11 Donc, dans ce cas, désir d'apprendre l'anglais et difficultés financières se conjuguent.

Pour d'autres, c'est la visite des évêques missionnaires qui fait se cristalliser une vocation latente. Ainsi l'abbé FrançoisJoseph Cénas, à la suite d'une conversation avec Mgr Demers, évêque de Vancouver, fait part à Mgr Bourget de son désir de partir pour l'Oregon, dans une lettre du 23 octobre 1849 . Il partira peu après. ${ }^{12}$

Une autre dimension nous est révélée par l'abbé L.-G. Plamondon. Faisant part de son désir d'aller en mission en Nouvelle-Angleterre, il dit ceci à Mgr Bourget, pour expliquer le fait qu'il a tant tardé à s'en ouvrir: "Je craignais que l'on me supposa un désir d'émancipation tel qu'on ose l'attribuer généralement à tous ceux qui veulent ainsi se consacrer pour un temps au salut de leurs compatriotes." 13 Ici, c'est donc le désir de promotion de certains autres qui nous est révélé.

Enfin, la santé ! À propos de l'abbé H.-O. Landry, le Dr A.-A. Roy, de Webster, écrit, le 26 octobre 1871: "En conséquence, mon opinion est qu'il est prudent et je pense même nécessaire que le Révérend $\mathrm{H}$. Landry vive sous un climat tempéré et qu'un climat trop froid le conduira invariablement à la consomption." ${ }^{14}$ Il restera aux Etats-Unis et vivra jusqu'en 1890 !

Ainsi, toute une variété de motifs se sont entremêlés amenant le départ de ces nombreux prêtres séculiers de Montréal.

11 ACAM, Dossier 421.049.

12 ACAM, Dossier 421.100.

13 ACAM, Dossier 421.254, Lettre du 21 septembre 1870.

14 ACAM, Dossier 421.257. 


\section{Motifs de Mgr Bourget}

Comme on a pu le constater, la part du clergé séculier du diocèse de Montréal, entre 1836 et 1876, a été d'une générosité surprenante envers les États-Unis. Car il faut compter qu'à la même époque l'Ouest canadien et les Provinces maritimes demandaient aussi à Montréal leur participation.

Comment cela se fait-il? Nous croyons que nous pouvons appliquer à l'Église des Etats-Unis ce que le chanoine Groulx disait des missions du Nord-Ouest canadien: "Parmi les auxiliaires de ces missions, le premier rang appartient toutefois aux évêques du Bas-Canada. Et, entre tous, accordons une mention spéciale à l'évêque de Montréal, Mgr Ignace Bourget. Il semble que cet homme, plus que personne, se tienne aux écoutes de tous les besoins, de toutes les misères de l'Église canadienne [ajoutons: et américaine]. Les missionnaires eux-mêmes s'adressent à lui comme au père qui entend toutes les prières. De cet évêque au grand cœur, d'un cœur aussi grand que son immense foi, on peut user et abuser comme l'on abuse d'un saint." 15

Un trait nous permettra de découvrir pourquoi. Le 17 novembre 1874, de Fort Totten, Dakota Territories, l'abbé L. Bonin écrit à Mgr Bourget: "Le 24 septembre au matin, avant mon départ de Montréal, Monsieur le Secrétaire me remettait de la part de Votre Grandeur, entre autres choses, une lettre d'instructions. Monseigneur, j'ai été profondément touché de vos avis paternels... Je les méditerai et je les reméditerai encore... Oh! Que Votre Grandeur a été bonne pour moi !" 16

Si tous les prêtres qui ont quitté Montréal ont été l'objet d'une sollicitude semblable et en ont été aussi touchés, il n'est pas surprenant qu'au moment des difficultés, devant les vastes besoins des Canadiens aux Etats-Unis ou des missions indiennes, ils se soient tournés vers celui qui les avait guidés. C'est de lui qu'ils ont espéré et reçu argent, secours et renforts en prêtres, religieux et religieuses. L'âme d'ardent apôtre de Mgr Bourget a trouvé là un champ de prédilection.

15 Chan. Lionel Groulx, Le Canada français missionnaire, 26.

16 ACAM, Dossier 421.282. 
Écoutons-le nous livrer sa pensée, dans une lettre adressée à l'abbé Augustin Laverdière, prêtre de Québec, missionnaire en Floride. ${ }^{17} \mathrm{La}$ lettre est du 11 janvier 1868: "Pour ma part, je suis heureux de vous voir plein de courage et animé du véritable dévouement, qui sacrifie tout et ne recule devant aucune difficulté quand il s'agit de prouver son amour pour la sainte Église, en se consumant de travaux pour la répandre et l'exalter dans les lieux les plus lointains. Notre Seigneur nous en donne l'exemple, qu'il accompagne de sa grâce toute puissante. "Le Christ a aimé l'Église et s'est livré pour elle [St-Paul]." 18 Voilà le motif profond de son attitude envers les missions américaines!

Chancellerie de l'archidiocèse

François Beaudin, ptre, Archiviste

de Montréal.

${ }^{17}$ Cet abbé Laverdière est le frère de l'abbé C.-H. Laverdière, éditeur des Oeuvres de Champlain, en 1870. $17: 116$

18 En latin, dans le texte. ACAM, Registres des Lettres de Mgr Bourget, 